\section{Efeito do exercício físico sobre peso corporal em crianças com excesso de peso: ensaio clínico comunitário randomizado em uma favela no Brasil}

\author{
Effect of physical exercise on bodyweight in \\ overweight children: a randomized controlled trial \\ in a Brazilian slum
}

\author{
${ }^{1}$ Instituto Materno Infantil \\ Professor Fernando Figueira, \\ Recife, Brasil. \\ 2 Medical Research Council \\ and Epidemiology Resource \\ Centre, University of \\ Southampton, Southampton, \\ $U . K$ \\ ${ }^{3}$ Medical Research Council \\ and Social \& Public Health \\ Sciences Unit, University of \\ Glasgow, Glasgow, U.K. \\ Correspondência \\ J. G. B. Alves \\ Instituto Materno Infantil \\ Professor Fernando Figueira. \\ Rua dos Coelhos 300 \\ Recife, $P E$ \\ 50070-550, Brasil. \\ joaoguilherme@imip.org.br
}

\begin{abstract}
Given the increase in obesity in developed and developing countries and its concomitant morbidity, successful treatment approaches are needed. We examined the effect of a structured exercise intervention in overweight children in a slum in Recife, Pernambuco State, Brazil. This was a randomized, controlled efficacy trial. Seventy-eight children were randomized. Exercise was supervised, consisting of three 50' group aerobics sessions per week for six months. All participants maintained ad libitum diets. Based on intention-to-treat analyses, children in both groups had a significant increase in weight at follow-up ( $p$-value for within-group increase $\leq$ 0.01). The increase in weight was significantly lower in the exercise group (mean difference between groups; -1.37; 95\%CI: -2.00; -0.74). A significant difference $(p=0.049)$ between the exercise and control groups at six-month followup was also found for BMI (mean difference between groups; -0.53; 95\%CI: -1.06; -0.002). When we restricted the analyses to children who completed the trial (intervention $=30$ and control $=$ $38)$, the results were the same. An exercise program for children, sustained for six months, was effective for reducing weight gain in overweight children living in a very poor neighborhood.
\end{abstract}

Nutritional Epidemiology; Obesity; Overweight; Physical Education and Training; Child
João Guilherme B. Alves 1

Catharine R. Galé 2

Edvaldo Souza 1

G. David Batty 3

\section{Introdução}

Nas últimas décadas, a obesidade tem se apresentado como uma epidemia global em crianças 1 . A obesidade e o sobrepeso estão fortemente associados com o aumento da morbidade na infância e na vida adulta 2 . Estudos transversais em crianças com excesso de peso demonstram uma associação com maior risco para doenças cardiovasculares, afecções ortopédicas, respiratórias, endócrinas, além de uma baixa auto-estima $2,3,4,5,6,7,8$. Os estudos de coorte em crianças comprovam que aquelas com peso mais elevado apresentam maior risco de permanecerem com excesso de peso na vida adulta, com maiores riscos de desenvolverem doença cardiovascular, hipertensão arterial, dislipidemia, aterosclerose, diabetes tipo 2, disfunção hepática e outras co-morbidades 9,10 .

O excesso de peso é considerado atualmente como uma afecção metabólica complexa e que resulta, fundamentalmente, do desequilíbrio entre a ingestão e o gasto calórico ${ }^{11}$. Diminuição das dietas energeticamente densas, mudanças dos hábitos sedentários e aumento da atividade física têm sido consideradas como as principais intervenções no controle de crianças com excesso de peso 12,13,14. Entretanto, intervenções como a prática de atividade física têm levado a resultados discordantes: alguns estudos randomizados têm demonstrado que a prática de incorporar exercícios físicos parece ser mais efetiva em pro- 
mover perda de peso a longo prazo em crianças com excesso de peso, do que aquela de apenas incorporar orientações dietéticas 15,16,17. Entretanto, não existe um consenso em relação a esses achados 18 .

A análise comparativa dos estudos envolvendo intervenções na área da atividade física é dificultada por algumas limitações metodológicas como: ausência de análise por intenção de tratamento, amostras subdimensionadas, curta duração do seguimento e ausência de padronização da intensidade da atividade física nos participantes dos estudos 16. Além do mais, grande parte do que é entendido sobre o papel potencial da atividade física, como modalidade de tratamento, é oriunda de estudos conduzidos em países desenvolvidos 17,18. Em nossa pesquisa bibliográfica, não encontramos estudos realizados nos países em desenvolvimento, com objetivos de verificar a eficácia de uma intervenção exclusiva com exercício físico para o controle de excesso de peso, sem a incorporação de orientações dietéticas, em crianças carentes. Foi esse, portanto, o objetivo do presente estudo.

\section{Métodos e sujeitos}

\section{Local e população}

O estudo foi realizado na favela de Caranguejo, localizada na Região Metropolitana do Recife, Pernambuco, Nordeste do Brasil. Essa comunidade apresentava uma população de 3.733 indivíduos no ano do início da pesquisa, 2005, sendo que mais de $90 \%$ deles tinham uma renda per capita inferior a um dólar americano por dia. A população elegível ao estudo consistiu das 638 crianças na faixa etária dos 5 aos 10 anos, residentes na favela e cadastradas no Programa Saúde da Família (PSF), desenvolvido pelo Instituto Materno Infantil Professor Fernando Figueira (IMIP) em conjunto com a Prefeitura Municipal da cidade do Recife (Figura 1).

Foram selecionadas as crianças que apresentavam: (a) índice de massa corporal (IMC) $\geq$ percentil 85; (b) idade entre cinco e dez anos; (c) ausência de evidência clínica de cardiopatia congênita ou adquirida, insuficiência respiratória, diabetes tipo 1; (d) não fazer uso de medicamentos que interfiram com a resposta cardíaca durante o exercício, como beta-bloqueadores. As crianças selecionadas à pesquisa foram alocadas ao grupo de intervenção ou controle, de forma randomizada. A randomização foi realizada usando-se o programa Epi Info, versão 6.04 (Centers for Disease Control and Prevention, Atlanta, Estados Unidos), módulo epitable. Inicialmente os pacientes foram listados consecutivamente, e após selecionados aleatoriamente, sem reposição, para compor o grupo da intervenção. Os demais, formaram o grupo controle. Não foi oferecida nenhuma orientação alimentar a nenhum dos participantes, assim como aos seus responsáveis.

\section{Programa de exercício físico}

O programa de exercício físico, independente das aulas curriculares normais das escolas públicas freqüentadas pelas crianças, teve sempre a duração de cinqüenta minutos e foi ministrado três vezes por semana durante um período de seis meses. As aulas foram administradas por alunos do curso de graduação em educação física da Universidade de Pernambuco (UPE), com a supervisão de uma professora de educação física, sendo constituídas por atividades recreativas com gasto energético de intensidade moderada (jogar bola, correr, pular, dançar). O grupo controle não participou desse programa de exercícios físicos.

\section{Avaliação antropométrica}

O peso e a estatura foram aferidos no início do estudo e seis meses após. A mensuração foi realizada com as crianças sem calçados e usando apenas um calção. A estatura foi medida por um estadiômetro montado com aproximação para $0,1 \mathrm{~cm}$. O peso foi tomado usando-se uma balança previamente calibrada com escala de aproximação para $0,1 \mathrm{~kg}$. O IMC foi calculado pela divisão do peso pela estatura elevada ao quadrado $\left(\mathrm{kg} / \mathrm{m}^{2}\right)$.

\section{Cálculo do tamanho da amostra e análise estatística}

A amostra foi calculada para se detectar uma diferença no IMC de $0,1 \mathrm{~kg} / \mathrm{m}^{2}$, com um poder estatístico de $80 \%$ e um erro alfa de 0,05 . Com esses parâmetros, foi determinado um número de 32 crianças por grupo. Para compensar as perdas, a amostra foi elevada em $10 \%$.

A análise para verificar o efeito da intervenção foi realizada utilizando-se o método por intenção de tratamento. As análises foram realizadas para todas as crianças, independente de terem freqüentado as aulas regularmente ou não. Também foi estimado o impacto da intervenção somente entre crianças que freqüentaram pelo menos $75 \%$ das aulas. Para detector se a randomização havia produzido grupos comparáveis, as características dos dois grupos foram comparadas antes da intervenção usando-se o teste $t$ 
de Student para amostras não pareadas e variáveis contínuas; o teste do $\chi^{2}$ foi empregado para as variáveis categóricas. $\mathrm{O}$ teste $\mathrm{T}$ para amostras não pareadas foi utilizado para comparar diferenças entre os grupos, peso e IMC em valores absolutos e percentuais antes da intervenção e no final do estudo. O teste t de Student para amostras pareadas foi realizado para determinar se havia diferenças significativas dentro de cada grupo, intervenção e controle. Foi adotado como significativo um valor de $\mathrm{p}<0,05$.

\section{Aspectos éticos}

Todos os pais forneceram o Termo de Consentimento Informado por escrito, e o projeto desta pesquisa foi previamente aprovado pelo Comitê de Ética em Pesquisa do IMIP. Todas as crianças, antes de serem admitidas à pesquisa, foram submetidas a uma avaliação médica com história e exame físico detalhados.

\section{Resultados}

Setenta e oito crianças foram inicialmente randomizadas para os grupos de intervenção e controle (Figura 1); 39 em cada. Nove crianças no grupo de intervenção e uma no grupo controle não concluíram o estudo. Não houve diferença entre os grupos, antes da intervenção, em relação a idade $(7,56 \pm 1,77$ anos versus $7,86 \pm 1,51 ; 0,677)$, peso $(36,1 \pm 13,4 \mathrm{~kg}$ versus $33,7 \pm 8,98 ; 0,571) \mathrm{e}$ IMC $\left(20,9 \pm 3,71 \mathrm{~kg} / \mathrm{m}^{2}\right.$ versus $\left.20,8 \pm 2,76 ; 0,533\right)$ entre os participantes que completaram ou não o estudo.

As características sócio-econômicas e biológicas dos participantes, de acordo com os grupos intervenção e controle, encontram-se na Tabela 1. Não houve diferença significativa entre os grupos em relação a idade, IMC, número de irmãos no domicílio, cor, freqüência à escola, renda per capita, anos de escolaridade materna, horas diárias gastas assistindo à TV e presença no domicílio de TV e refrigerador. Foi observada uma pequena diferença em relação ao IMC ( $\mathrm{p}=0,048)$.

Na Tabela 2, são apresentados o peso e o IMC, antes da intervenção e no final do seguimento, de acordo com o grupo de alocação; os dados foram analisados segundo a base por intenção de tratamento. As crianças em ambos os grupos apresentaram um significativo aumento de peso durante os seis meses do estudo (valor de p para cada grupo $\leq 0,01$ ). Esse aumento foi menor no grupo da intervenção quando comparado ao controle (diferença média entre os grupos; -1,37; IC95\%: -2,00; -0,74).
Figura 1

Diagrama do fluxo das crianças participantes do ensaio.

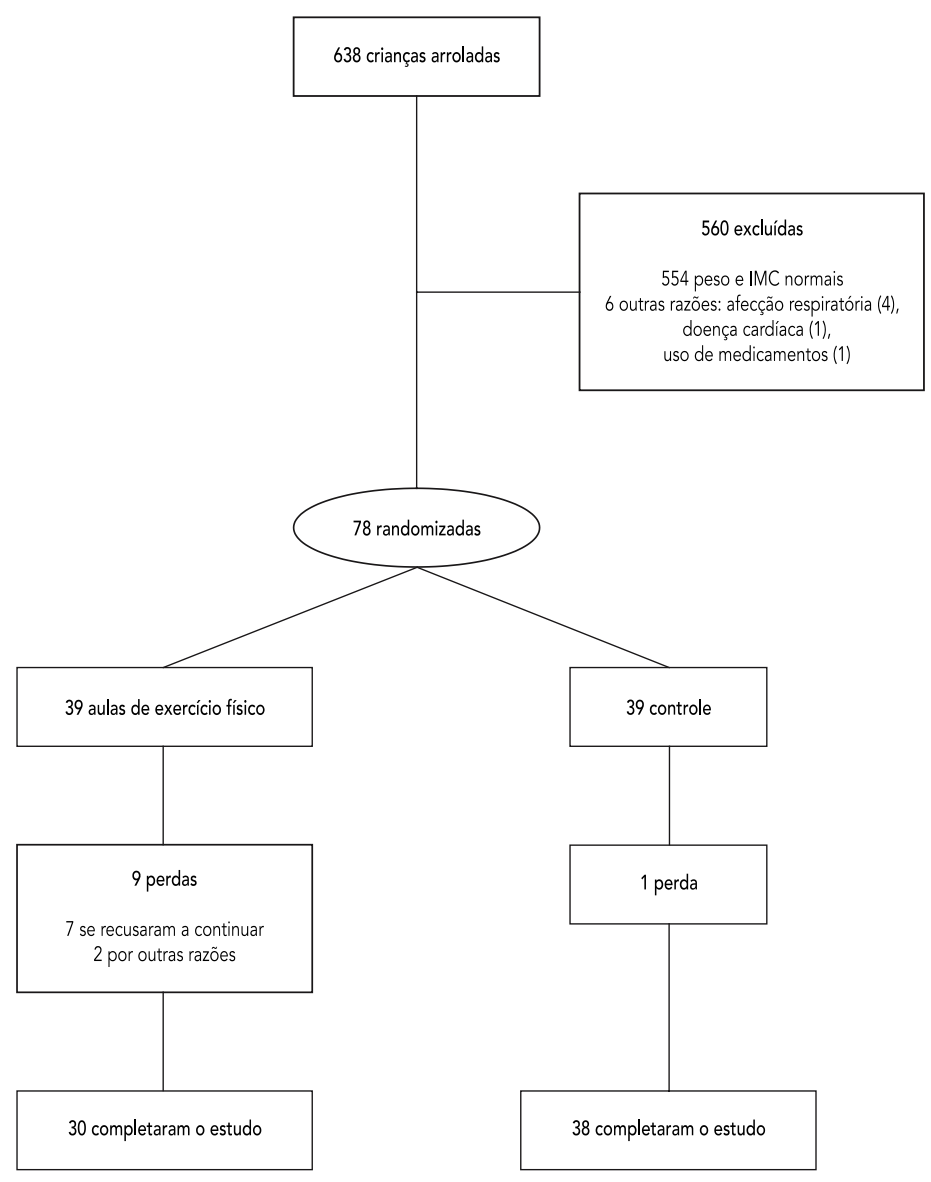

IMC: índice de massa corporal.

Não houve diferença significativa dentro dos grupos em relação ao IMC antes da intervenção e no final do seguimento (Tabela 2). Entretanto, o declínio do IMC no grupo de intervenção resultou em uma diferença significativa $(p=0,049)$ entre os dois grupos, após os seis meses de seguimento (diferença média entre os grupos; -0,53; IC95\%: -1,06; -0,002).

Setenta e sete por cento dos participantes no grupo de intervenção completaram os seis meses do programa de exercícios físicos; no grupo controle, foram acompanhados $97,5 \%$ dos participantes. Quando restringimos a análise para as crianças que completaram o estudo, os resultados foram essencialmente os mesmos dos relatados acima. 
Características na linha de base dos 78 participantes do estudo na Favela do Caranguejo, Recife, Pernambuco, Brasil, 2006.

\begin{tabular}{|c|c|c|c|}
\hline & $\begin{array}{c}\text { Grupo intervenção }(n=39) \\
\text { Média }(D P) \text { * }\end{array}$ & $\begin{array}{c}\text { Grupo controle }(n=39) \\
\text { Média }(D P) \text { * }\end{array}$ & Valor de $\mathrm{p}$ \\
\hline Idade (anos) & $7,97(1,81)$ & $7,85(1,47)$ & 0,733 \\
\hline Peso (kg) & $35,4(12,3)$ & $34,4(9,75)$ & 0,684 \\
\hline Estatura (m) & $1,30(0,13)$ & $1,27(0,12)$ & 0,260 \\
\hline IMC (kg/m²) & $20,6(3,33)$ & $21,0(2,90)$ & 0,497 \\
\hline Número de irmãos & $1,49(1,00)$ & $1,51(1,07)$ & 0,913 \\
\hline \multirow[t]{2}{*}{ Horas assistindo à TV/dia } & $3,51(1,27)$ & $3,64(0,71)$ & 0,584 \\
\hline & $\mathrm{n}(\%)$ ** & n (\%) ** & \\
\hline \multicolumn{4}{|l|}{ Sexo } \\
\hline Masculino & $18(46,2)$ & $22(56,4)$ & 0,365 \\
\hline Feminino & $21(53,8)$ & $17(43,6)$ & \\
\hline \multicolumn{4}{|l|}{ Cor } \\
\hline Branca & $19(48,7)$ & $20(51,3)$ & 0,962 \\
\hline Preta & $10(25,6)$ & $10(25,6)$ & \\
\hline Mestiço & $10(25,6)$ & $9(23,1)$ & \\
\hline \multicolumn{4}{|l|}{ TV em casa } \\
\hline Sim & $37(94,9)$ & $38(97,4)$ & 0,556 \\
\hline Não & $2(5,1)$ & $1(2,6)$ & \\
\hline \multicolumn{4}{|l|}{ Freqüenta a escola } \\
\hline Sim & $34(87,2)$ & $36(92,3)$ & 0,455 \\
\hline Não & $2(5,1)$ & $1(2,6)$ & \\
\hline \multicolumn{4}{|l|}{ Refrigerador em casa } \\
\hline Sim & $33(84,6)$ & $33(84,6)$ & 1,00 \\
\hline Não & $6(15,4)$ & $6(15,4)$ & \\
\hline \multicolumn{4}{|c|}{ Anos de escolaridade materna } \\
\hline$\geq 4$ & $24(61,5)$ & $24(61,5)$ & 1,00 \\
\hline$<4$ & $15(38,5)$ & $15(38,5)$ & \\
\hline \multicolumn{4}{|l|}{ Renda per capita/dia (US\$) } \\
\hline$<1,0$ & $28(71,8)$ & $28(71,8)$ & 1,00 \\
\hline$\geq 1,0$ & $11(28,2)$ & $11(28,2)$ & \\
\hline
\end{tabular}

* teste t de Student;

** Qui-quadrado.

\section{Discussão}

O objetivo deste ensaio foi verificar a eficácia de uma determinada quantidade de atividade física na promoção da perda de peso em crianças com excesso de peso ou obesidade. O principal achado do estudo foi o de que a prática de exercício físico regular por seis meses, sem a necessidade de uma intervenção dietética, para crianças que vivem em situação de risco alimentar pelas precárias condições sócio-econômicas, resulta numa significativa redução do IMC e em um menor ganho ponderal. Para o nosso conhecimento, este é o primeiro estudo, ensaio comunitário randomizado e controlado, a examinar o impacto de uma intervenção com exercício físico para o tratamen- to de crianças portadoras de excesso de peso, realizado em um país em desenvolvimento.

A perda de peso ao final dos seis meses de estudo foi estatisticamente significativa, apesar de modesta, em termos absolutos. Esses achados são consistentes com alguns estudos realizados em adultos usando-se apenas intervenção com exercício físico de intensidade de moderada a vigorosa, sem alterações na dieta 19. Epstein \& Goldfield 16 afirmam que o exercício físico parece desempenhar um efeito sinérgico com as alterações dietéticas na promoção da perda de peso, apesar do pequeno número de pesquisas envolvendo a eficácia do exercício físico isolado no tratamento do excesso de peso em adultos e especialmente em crianças. Um estudo realizado 
Peso, estatura e índice de massa corporal (IMC) na linha de base e seis meses após nos grupos de intervenção e controle, Favela do Caranguejo, Recife, Pernambuco, Brasil, 2006.

\begin{tabular}{lccc}
\hline & $\begin{array}{c}\text { Grupo intervenção } \\
(\mathbf{n}=39)\end{array}$ & $\begin{array}{c}\text { Grupo controle } \\
(\mathbf{n}=39)\end{array}$ & $\begin{array}{c}\text { Diferença entre os grupos } \\
\text { Média (IC95\%) }\end{array}$ \\
\hline Valor de $\mathbf{p}$
\end{tabular}

na China observou em crianças, na idade escolar, que a prática de exercício físico de intensidade moderada ou vigorosa foi eficaz no combate ao ganho de peso excessivo 20 . Reilly \& McDowell 15 apontam para evidências de que o aumento da atividade física seja uma intervenção eficaz no tratamento da obesidade. Ao lado do efeito na redução do peso, programas regulares de exercícios físicos contribuem para restaurar a disfunção endotelial provocada pela obesidade, além de contribuir para a melhora da aptidão física das crianças obesas 21 .

O fato dos dois grupos em nosso estudo terem apresentado ganho ponderal se justifica por termos estudado crianças, em fase de crescimento, em que nesta faixa etária se espera um ganho ponderal médio de $1,5 \mathrm{~kg}$ e um incremento de estatura por volta de $3 \mathrm{~cm}$ no semestre 22 . Observamos que apesar do incremento de peso nos dois grupos no período estudado, essas alterações foram diferentes em relação aos seus percentuais e números absolutos. O mesmo raciocínio se aplica aos valores do IMC. Como inclusive detectamos uma diminuição do IMC no grupo estudo, podemos admitir que este Índice seja mais sensível do que o peso, isoladamente, para avaliar resultados deste tipo de intervenção, uma vez que o IMC avalia concomitantemente os dois parâmetros, peso e estatura, ambos em fase de crescimento na faixa etária aqui estudada.
Nos países em desenvolvimento, como o Brasil, o aumento da prevalência do excesso de peso e de suas complicações associadas, representa um importante desafio ao setor saúde, não só pelo crescimento da morbidade, tanto na infância como na vida adulta, acarretando perda de anos saudáveis de vida e forte impacto econômico 23,24. Pelos achados deste estudo, parece possível que um programa de exercícios físicos regulares, direcionado às populações carentes, que no Brasil, atualmente, vem apresentando uma maior incidência de excesso de peso, quando comparada às populações abastadas, possa contribuir diretamente para um melhor controle das afecções crônicas que têm o excesso de peso como importante fator de risco.

Paralelamente ao aumento do excesso de peso, a análise do quadro atual epidemiológico da atividade física no Brasil é preocupante. Quando comparada aos países desenvolvidos, a atividade física de lazer praticada por adultos é bem inferior, apesar de apresentar a mesma descrição de um maior nível entre os homens 25,26. Entretanto, o tema vem atualmente recebendo mais atenção dos pesquisadores. Recente revisão sistemática observou uma tendência de aumento de publicações a partir de 2000, embora com disparidades regionais, havendo concentração de estudos nas regiões Sudeste e Sul. A maioria dos 42 estudos incluídos utilizou questionários como instrumentos de pesquisa, cujos conteúdos variaram, 
dificultando a comparação entre eles, tornando a padronização de instrumentos e definições, essenciais para o avanço científico da área 27 .

Nosso estudo, apesar de ter tido um desenho randomizado e controlado, focalizando uma população sócio-economicamente marginalizada e que sobrevive em uma situação de risco alimentar, apresenta algumas limitações metodológicas. Inicialmente, aferimos apenas o peso e o IMC, o que necessariamente não traduzem adiposidade; algumas dessas crianças podem ter apresentado variações no peso ou IMC por modificações da massa muscular, resultantes do exercício físico. Também desconhecemos a duração do efeito dessa intervenção após a sua interrupção, uma vez que não acompanhamos a evolução ponderal e do IMC depois da suspensão dos seis meses da intervenção proposta, sendo este o objetivo de um novo estudo. Di-

\section{Resumo}

Associação da obesidade com doenças crônicas tem se mostrado mais intensa nas áreas carentes. Examinamos o efeito de um programa de exercício físico para crianças com excesso de peso, em uma favela do Recife, Pernambuco, Brasil, por meio de um ensaio comunitário, randomizado, com 78 crianças. Um grupo $(n=39)$ recebeu três aulas semanais de exercícios físicos durante seis meses. Não foi realizada nenhuma intervenção em relação à alimentação. A análise por intenção de tratamento demonstrou que todas as crianças apresentaram aumento significativo de peso. Entretanto, esse aumento foi menor no grupo que sofreu a intervenção (diferença média entre os grupos; -1.37; IC95\%: -2,00; -0,74). Em relação ao índice de massa corporal (IMC), também foi verificada uma diferença significativa ( $p=0,049)$ entre os dois grupos (diferença média entre os grupos; -0,53; IC95\%: -1,06; -0,002). Na análise restrita às crianças que completaram o estudo (interven$c ̧ \tilde{a} o=30$ e controle $=38$ ), os resultados foram similares. Concluímos que um programa regular de exercícios físicos para crianças com excesso de peso em áreas carentes seja eficaz, sem a necessidade de intervenções dietéticas, na redução do ganho ponderal e do IMC.

Epidemiologia Nutricional; Obesidade; Excesso de Peso; Educação Física e Treinamento; Criança ferentemente de estudos realizados em países desenvolvidos, não acreditamos que em nossa população a simples prática de exercícios físicos regulares possa ter modificado os hábitos dietéticos, especialmente pela situação de risco alimentar em que essas pessoas sobrevivem e também pelo baixo nível de instrução dos pais. Apesar disso, não podemos deixar de registrar que o não controle da alimentação dos participantes foi uma limitação do trabalho.

Em conclusão, nosso estudo fornece subsídios para o desenvolvimento de programas de intervenção com aulas estruturadas de exercícios físicos, administradas de forma regular e mantidas por um período de seis meses, como uma forma eficaz no controle de peso e IMC em crianças com excesso de peso residentes em uma área de baixa renda, sem a necessidade de orientação dietética.

\section{Colaboradores}

J. G. B. Alves elaborou o projeto e coordenou as fases de implantação, análise dos dados e foi co-autor na redação final do estudo, também realizou a tradução final da versão da língua inglesa para o português. C. R. Galé desenvolveu a estratégia para a análise dos dados; foi responsável por toda a análise estatística e co-autora da redação final do estudo. E. Souza comentou todas as versões do manuscrito. G. D. Batty desenvolveu a análise estratégica e foi co-autor da redação final do estudo. 


\section{Referências}

1. Kosti RI, Panagiotakos DB.The epidemic of obesity in children and adolescents in the world. Cent Eur J Public Health 2006; 14:151-9.

2. Must A, Strauss RS. Risks and consequences of childhood and adolescent obesity. Int J Obes Relat Metab Disord 1999; 23 Suppl 2:S2-11.

3. Botton J, Heude B, Ketanneh A, Borys JM, Lommez A, Bresson JL, et al. Cardiovascular risk factor levels and their relationships with overweight and fat distribution in children: the Fleurbaix Laventie Ville Sante II study. Metabolism 2007; 56:614-22.

4. Taylor ED, Theim KR, Mirch MC, Ghorbani S, Tanofsky-Kraff M, Adler-Wailes DC, et al. Orthopedic complications of overweight in children and adolescents. Pediatrics 2006; 117:2167-74.

5. Boran P, Tokuc G, Pisgin B, Oktem S, Yegin Z, Bostan O. Impact of obesity on ventilatory function. J Pediatr (Rio J) 2007; 83:171-6.

6. Williams J, Wake M, Hesketh K, Maher E, Waters E. Health-related quality of life of overweight and obese children. JAMA 2005; 293:70-6.

7. Kaur S, Kapil U, Singh P. Pattern of chronic diseases amongst adolescent obese children in developing countries. Curr Sci 2005; 88:1052-6.

8. Reilly JJ, Methven E, McDowell ZC, Hacking B, Alexander D, Stewart L, et al. Health consequences of obesity. Arch Dis Child 2003; 88:748-52.

9. Cheung YB, Machin D, Karlberg J, Khoo KS. A longitudinal study of paediatric BMI values predicted health in middle age. J Clin Epidemiol 2004; 57:1316-22.

10. Olsen LH, Baker JL, Holst C, Sorensen TL. Birth cohort effect on the obesity epidemic in Denmark. Epidemiology 2006; 17:292-5.

11. Speiser P, Rudolf MCJ, Anhalt H, Camacho-Hubner C, Chiarelli F, Eliakim A, et al. Consensus development: childhood obesity. J Clin Endocrinol Metab 2005; 90:1871-87.

12. Harsha DW. The benefits of physical activity in childhood. Am J Med Sci 1995; 310 Suppl 1: S109-13.

13. Kennedy E, Goldberg J. What are American children eating? Implications for public policy. Nutr Rev 1995; 53:111-26.

14. Flodmark CE, Lissau I, Moreno LA, Pietrobelli A, Widhalm K. New insights into the field of children and adolescents' obesity: the European perspective. Int J Obes 2004; 28:1189-96.

15. Reilly JJ, McDowell ZC. Physical activity interventions in the prevention and treatment of paediatric obesity: systematic review and critical appraisal. Proc Nutr Soc 2003; 62:611-9.
16. Epstein LH, Goldfield GS. Physical activity in the treatment of childhood overweight and obesity: current evidence and research issues. Med Sci Sports Exerc 1999; 31(11 Suppl):S553-9.

17. Snethen JA, Broome ME, Cashim SE. Effective weight loss for overweight children: a meta-analysis of intervention studies. J Pediatr Nurs 2006; 21:45-56.

18. Atlantis E, Barnes EH, Singh MA. Efficacy of exercise for treating overweight in children and adolescents: a systematic review. Int J Obes (Lond) 2006; 30:1027-40.

19. Wing RR. Physical activity in the treatment of the adulthood overweight and obesity: current evidence and research issues. Med Sci Sports Exerc 1999; 31(11 Suppl):S547-52.

20. Li L, Li K, Ushijima H. Moderate-vigorous physical activity and body fatness in Chinese urban school children. Pediatr Int 2007; 49:280-5.

21. Meyer AA, Kundt G, Lenschow U, Schuff-Werner P, Kienast W. Improvement of early vascular changes and cardiovascular risk factors in obese children after a six-month exercise program. J Am Coll Cardiol 2006; 48:1865-70.

22. Needlman RD. Growth and development. In: Behrman RE, Kliegman RM, Jenson HB, editors. Nelson textbook of pediatrics. 16 th Ed. Philadelphia:W. B. Saunders Co.; 2000. p. 23-61.

23. Batista Filho M, Rissin A. A transição nutricional no Brasil: tendências regionais e temporais. Cad Saúde Pública 2003; 19 Suppl 1:S181-91.

24. Sichieri R, Nascimento S, Coutinho W. The burden of hospitalization due to overweight and obesity in Brazil. Cad Saúde Pública 2007; 23:1721-7.

25. Monteiro CA, Conde WL, Matsudo SM, Matsudo VR, Bonseñor IM, Lotufo PA. A descriptive epidemiology of leisure-time physical activity in Brazil, 1996-1997. Rev Panam Salud Pública 2003; 14: 246-54.

26. Dias-da-Costa JS, Hallal PC, Wells JC, Daltoé T, Fuchs SC, Menezes AM, et al. Epidemiology of leisure-time physical activity: a population-based study in southern Brazil. Cad Saúde Pública 2005; 21:275-82.

27. Hallal PC, Dumith SC, Bastos JP, Reichert FF, Siqueira FV, Azevedo MR. Evolution of the epidemiological research on physical activity in Brazil: a systematic review. Rev Saúde Pública 2007; 41:453-60.

Recebido em 08/Ago/2007

Versão final reapresentada em 23/Jan/2008

Aprovado em 28/Jan/2008 